\title{
Total Anomalous Pulmonary Venous Connection in Children
}

\author{
Amalia Făgărășan', Iolanda Muntean', Liliana Gozar', Sorina Pasc², Rodica Togănel'1 \\ 1 University of Medicine and Pharmacy, Tîrgu Mureș, Romania \\ 2 Clinic of Pediatric Cardiology III, Institute of Cardiovascular Diseases and Emergency Transplant, Tîrgu Mureș, Romania
}

\section{CORRESPONDENCE}

\section{Amalia Făgărășan}

Str. Gheorghe Marinescu nr. 50

540136 Tîrgu Mureș, Romania

Tel: +40 744967139

E-mail: amalia_fagarasan@yahoo.com

\section{ARTICLE HISTORY}

Received: 17 October, 2016

Accepted: 7 November, 2016
Iolanda Muntean • Str. Gheorghe Marinescu nr. 38 540142 Tîrgu Mureș, Romania Liliana Gozar - Str. Gheorghe Marinescu nr. 38, 540142 Tîrgu Mureș, Romania

Sorina Pasc • Str. Gheorghe Marinescu nr. 50, 540136 Tîrgu Mureș, Romania

Rodica Togănel • Str. Gheorghe Marinescu nr. 38 540142 Tîrgu Mures, Romania

\begin{abstract}
Introduction: The aim of this study was to study the anatomical types of total anomalous pulmonary venous connection (TAPVC), the associated cardiac and extracardiac congenita malformations, clinical manifestations, and postoperative evolution. Materials and methods: Twenty-four patients with a mean age of 125 days, admitted to the Clinic of Pediatric Cardiology III between January 1, 2009 and December 31, 2015 and diagnosed with TAPVC were included in the study. The patients were evaluated clinically, electrocardiographically and echocardiographically, both pre- and postoperatively. Postoperative evolution was monitored at 1, 3, 6, 12 and 24 months. Results: The anatomical types of TAPVC were: supracardiac in $50 \%$ of cases (12 patients), cardiac in $37.5 \%$ (9 patients) and mixed type in $12.5 \%$ of cases (3 patients). The first clinical manifestation was cyanosis in $72.2 \%$ of cases. Surgical correction was performed at a mean age of 37 days in obstructive forms, and 254 days in non-obstructive forms. From the study lot, $8.4 \%$ of patients had associated extracardiac malformations (anorectal agenesis and Ivemark syndrome). Early postoperative complications included pulmonary hypertension crisis (60\% of cases), supraventricular arrhythmias (35\% of cases) and chylothorax $(8.4 \%$ of cases). Late postoperative complications included: reintervention in $8 \%$ of patients with mixed type TAPVC. Conclusions: The most frequently encountered type was supracardiac TAPVC, which had a favorable postoperative evolution. Mixed type TAPVC had the highest rate of reintervention.
\end{abstract}

Keywords: total anomalous pulmonary venous connection, children, postoperative evolution

\section{INTRODUCTION}

Total anomalous pulmonary venous connection (TAPVC) is a rare form of congenital heart disease (CHD), characterized by the abnormal opening of the pulmonary veins either into one of the systemic veins (connection abnormality) or the atrium (drainage abnormality). Its prevalence is estimated at $0.6-1.2$ per 10,000 live births, being one of the most common causes of cyanosis in children with CHD. ${ }^{1,2}$ Thirty percent of TAPVC cases are associated with other complex heart defects. An atrial septal defect constitutes the pathway through which the left heart is supplied with blood, thus becoming an essential hemodynamic situation that ensures survival. Obstructive forms are neonatal cardiovascular emergencies that lead to death in the absence of surgical correction, ${ }^{3}$ which is 
often difficult and must be adapted to the anatomical type of TAPVC. ${ }^{4,5}$ The aim of this study was to analyze the anatomical subtypes, early clinical manifestations, as well as the early and late postoperative evolution of pediatric patients with TAPVC.

\section{MATERIAL AND METHODS}

Twenty-four patients with a mean age of 125 days (range 5 days - 15 years), admitted to the Clinic of Pediatric Cardiology III of Tîrgu Mureș between January 1, 2009 and December 31, 2015 and diagnosed with TAPVC were included in the study. Pre- and postoperative clinical and echocardiographic assessment was carried out in all patients. The echocardiographic evaluation was performed using a Philips IE 33x (PHILIPS IE-33 X MATRIX USA PHILIPS Product Ultrasound Echocardiography System) echocardiograph. The surgical procedures were carried out in the Clinic of Cardiovascular Surgery within the Cardiovascular Disease and Transplant Institute of Tîrgu Mureş. The demographic data, clinical manifestations and echocardiographic descriptions used in this observational retrospective study were obtained from the patients' observation records. Surgical procedures were described in the protocol registry of the Department of Cardiovascular Surgery. Early postoperative complications in patients being admitted to the pediatric Intensive Care Unit (ICU) were obtained from the epicrisis of transfer records. The diagnosis was made echocardiographically and in 6 cases it was augmented by computed tomography. The analysis of TAPVC type and associated congenital heart defects was made using echocardiography protocols. Inclusion criteria: age between $0-18$ years with simple or complex TAPVC (associated with various forms of congenital heart defects). Exclusion criteria: children with other types of congenital heart defects. Microsoft Excel 2007 was used for data centralization and statistical analysis was performed using the Student's t-test.

\section{RESULTS}

From the 24 children included in the study, $67 \%$ were males. Regarding age, $43 \%$ of the diagnosed children were newborns (0-29 days), 4\% were infants (1-3 months) and $53 \%$ were over 4 months old. Most patients (63\%) came from an urban background, and their weights varied from 2.2 to $3.8 \mathrm{~kg}$ (the mean weight at birth was $3.2 \mathrm{~kg}$ ). Clinical signs at admission included: cyanosis $(72.2 \%)$, feeding fatigue (33.6\%), poor weight gain (29.4\%), and diaphoresis $(8.4 \%)$. The analysis of the anatomical types of TAPVC showed: supracardiac type in $50 \%$ of cases $(n=12)$, cardiac type in $38 \%$ ( $n=9$, from which in 8 patients there was a connection to the coronary sinus, and one patient had direct drainage to the right atrium) and mixed type in $12 \%$ of patients $(n=3)$. Obstructive TAPVC was encountered in $21 \%$ of patients (4 with supracardiac and 1 with mixed type). Preoperative oxygen saturation levels were between $69 \%$ and $88 \%$ (mean value $78 \%$ ).

From the total number of patients included in the study, $8.4 \%$ had associated extracardiac congenital defects ( 1 case with anorectal agenesis and 1 case with supernumerary renal arteries and asplenia). Congenital heart defects associated with TAPVC are shown in Figure 1. The mean age at which surgical correction was performed was 37 days for obstructive TAPVC and 254 days for non-obstructive TAPVC. Pulmonary hypertension crisis occurred as an early postoperative complication in $60 \%$ of cases. Correlations between cardiopulmonary bypass

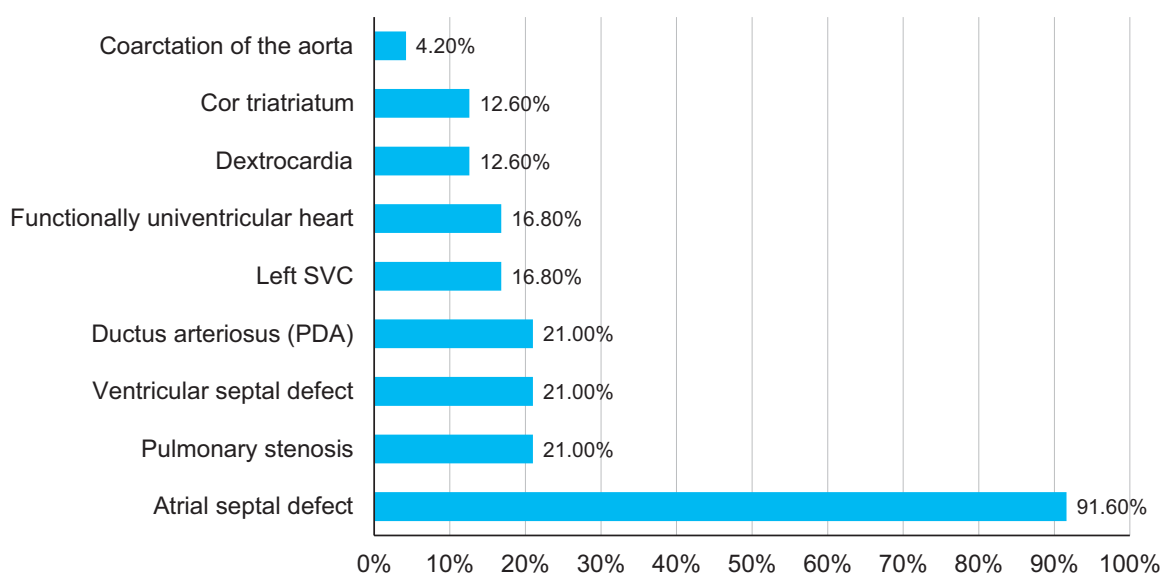

FIGURE 1. Congenital heart diseases associated with TAPVC. SVC - superior vena cava, PDA - patent ductus arteriosus 


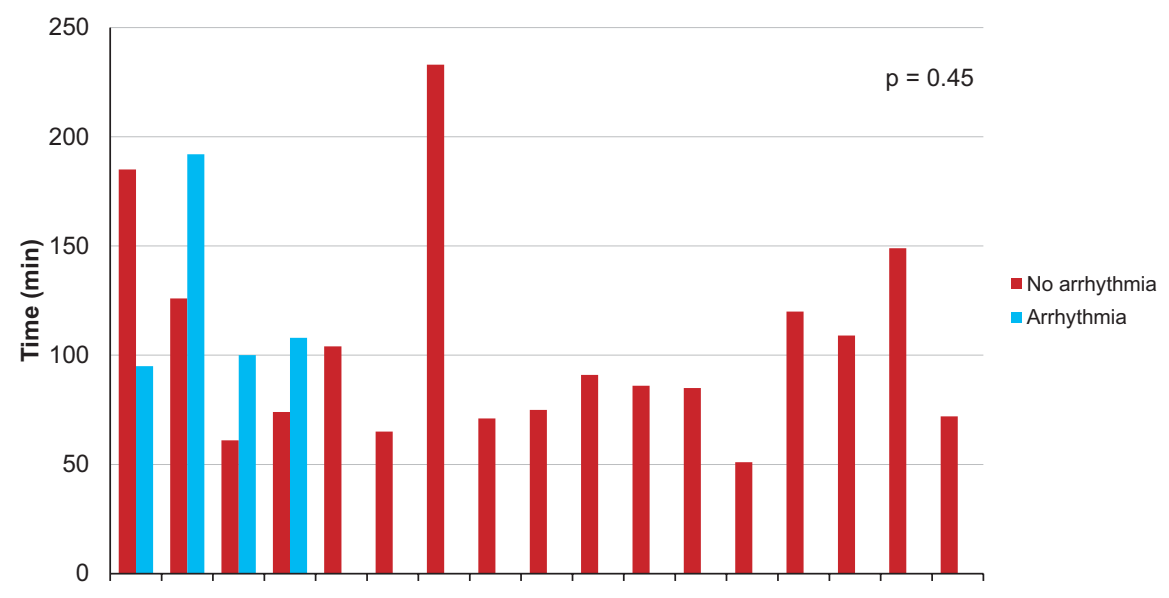

FIGURE 2. Bypass time in patients with TAPVC

time, cross-clamp time and risk of arrhythmias are shown in Figures 2 and 3.

The mean duration of preoperative care was 9 days (range 0 to 33 days), and the mean duration of hospitalization in the Intensive Care Unit was 5 days (range 1 to 22 days). There were two deaths in our study population: one patient with mixed type TAPVC (coronary sinus and left innominate vein) died postoperatively, and one patient with mixed type TAPVC (with infracardiac and supracardiac connection) died before the operation.

\section{DISCUSSION}

TAPVC is a cyanotic congenital heart disease, and its obstructive forms are considered neonatal emergencies. , $^{3,6}$ This study aimed to analyze the demographic characteristics, diagnosis and presentation of TAPVC, as well as its anatomical subtypes and postoperative evolution.

\section{Demographic characteristics}

TAPVC was predominantly encountered in males (67\%) in concordance with data presented in previous studies.2,7-9

\section{Diagnosis and presentation of TAPVC}

Early clinical manifestations included: cyanosis in $72.2 \%$ of cases, poor feeding in $33.6 \%$ of cases, reduced weight index in $29.4 \%$ of cases and diaphoresis in $8.4 \%$ of cases. Seale et al. published a study conducted on 422 patients, which showed cyanosis as the main early symptom in TAPVC. ${ }^{2}$

\section{Anatomical subtypes}

In establishing a diagnosis and determining the anatomical subtype, echocardiography is still the most useful noninvasive method. ${ }^{9,10}$ If the echocardiographic window is too

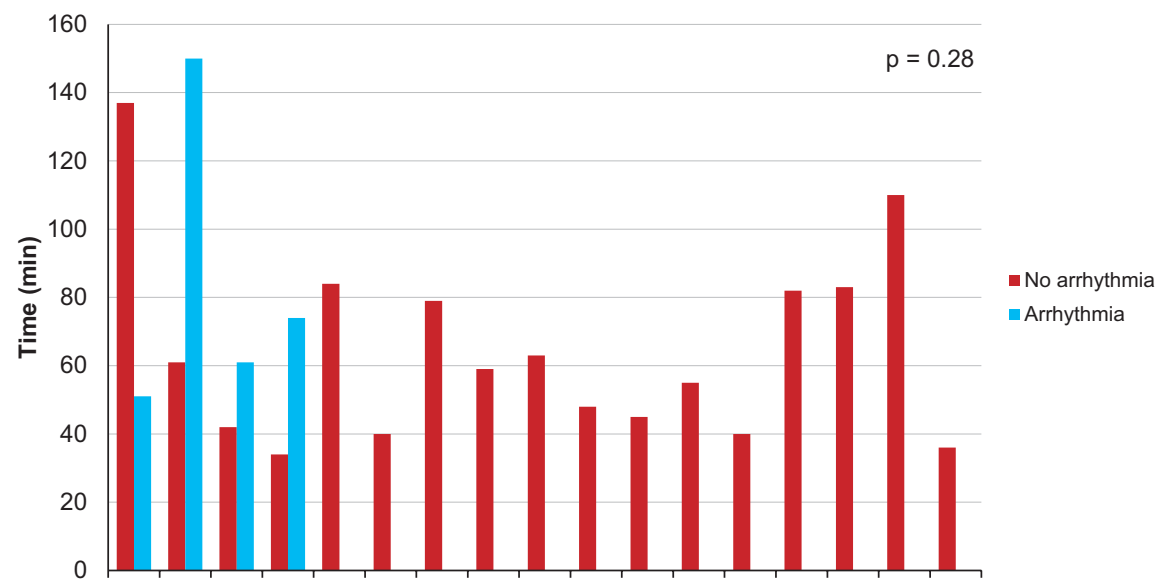

FIGURE 3. Cross-clamp time in patients with TAPVC 
narrow or the diagnosis is incomplete, especially in mixed or infracardiac types, MRI or multi-slice CT is required. ${ }^{11,12}$ Anatomical subtype was established using echocardiography, while in 6 cases CT was required. The Darling classification remains the most widely used method to classify TAPVC: subtype I - supracardiac anomalous pulmonary venous connection; subtype II - coronary sinus or direct right atrium anomalous pulmonary venous connection; subtype III - infracardiac anomalous pulmonary venous connection; subtype IV - unknown or multi-level anomalous pulmonary venous connection. ${ }^{13}$ According to other studies, mixed type is the most rarely seen TAPVC, in concordance with our own results. ${ }^{8,9}$ Complex forms of TAPVC, encountered in 4 patients, were associated with heterotaxy syndrome and single ventricle physiology, in concordance with studies concerning surgical approach and postoperative course. ${ }^{14,15}$ The diagnosis was established at a mean age of 125 days, later than in similar studies. $2,3,10,16$

\section{Postoperative evolution}

The mean age at which the patients benefited from surgical correction was 37 days in obstructive forms and 254 days in non-obstructive forms, also later than in similar studies due to the lack of a territorial network of pediatric cardiology services. ${ }^{3,8,17,18}$ Early postoperative complications included: pulmonary hypertension crisis in $60 \%$ of cases, which had improved after inhaling nitric oxide, supraventricular arrhythmias in 35\% of cases, laryngeal palsy and chylothorax in $12 \%$ of cases. Several studies show a correlation between the type of surgery performed and the risk of postoperative complications..$^{16,19-21}$ In our study, intraoperative variables such as cardiopulmonary bypass time and cross-clamp time were not associated with a higher risk of arrhythmias ( $p>0.05)$. Late postoperative complications occurred in 2 patients $(8 \%)$, at 9 and 12 months after the initial surgical procedure, in the form of obstruction. Both cases had mixed type TAPVC. Comparing the occurrence of postoperative arrhythmia with similar studies, we observed a lower incidence of sinus node dysfunction in survivors with TAPVC, namely $8 \%$ of cases ( 2 of 24). . $^{18,22}$

\section{Limitations}

This is a retrospective descriptive study on a small number of patients; however, TAPVS is a rare disease, therefore it can be difficult to collect an increased number of patients for a larger study lot.

\section{CONCLUSION}

The most frequently encountered type of TAPVC was the supracardiac type, which showed favorable early and late postoperative evolution, while mixed type TAPVC had the highest rate of reintervention.

\section{CONFLICT OF INTEREST}

The authors declare that there is no conflict of interests regarding the publication of this paper

\section{REFERENCES}

1. Reller MD, Strickland MJ, Riehle-Colarusso T, Mahle WT, Correa A Prevalence of congenital heart defects in metropolitan Atlanta, 19982005. J Pediatr. 2008:153:807-813

2. Seale AN, Uemura $H$, Webber SA, et al. Total Anomalous Pulmonary Venous Connection-Morphology and Outcomes From an International Populational-Base Study. Circulation. 2010;122:2718-2726.

3. Jinghao Z, Botao G, Zhiwei X, Jinfeng L. The Research on Operation of Obstructed Total Anomalous Pulmonary Venous Connection in Neonates. Scientific World Journal. 2014;2014:576569.

4. Kirshborn P, Jaggers J, Underleider R. Total anomalous pulmonary venous connection. In: Mavroudis C, Editor: Pediatric Cardiac Surgery. 3rd ed. Philadelphia: Mosby; 2003 p. 612-615.

5. Adzamli Kwashie I, Gaikwad S, Mali S, et al. Experience with the superior approach (Tucker's repair) for repair of supracardiac total anomalous pulmonary venous connection (TAPVC). Indian J Thorac Cardiovasc Surg. 2016;32:12.

6. Warrier G, Sasi Dharan B, Koshy S, et al. Repair of total anomalous pulmonary venous connection in neonates. The Journal of Thoracic and Cardiovascular Surgery. 2004;20:155-158.

7. St-LouisJ D, Harvey BA, Menk JS, et al. Repair of Simple Total Anomalous Pulmonary Venous Connection: A Review From the Pediatric Cardiac Care Consortium. Ann Thorac Surg. 2012;94:133-138.

8. Hancock Friesen CL, Zurakowski D, Thiagarajan RR, et al. Total anomalous pulmonary venous connection: An analysis of current management strategies in a single institution. Ann Thorac Surg. 2005;79:596-606;

9. Furlanetto $\mathrm{G}$, Furlanetto $\mathrm{BH}$, Henriques $\mathrm{SR}$, et al. Mixed type total anomalous pulmonary venous connection: early results and surgical techniques. World J Pediatr Congenit Heart Surg. 2015;6:26-32.

10. Eidem BW, Cetta F, et al. Capt 5: Anomalies of the pulmonary and systemic venous conections in Echocardiography in Pediatric and Adult Congenital Heart Diseases. Lippincott Williams \& Wilkins; 2010 p. 70-87.

11. Choe YH, Lee HJ, Kim HS, Ko JK, Kim JE, Han JJ. MRI of total anomalous pulmonary venous connections. J Comput Assist Tomogr. 1994;18:243-249.

12. Shiraishi I. Applications of multislice computed tomography imaging in children with congenital heart diseases. Kyobu Geka. 2007;60:619-626.

13. Craig JM, Darling RC, Rothney WB, et al. Total pulmonary venous drainage into the right side of the heart: report of 17 autopsied cases not associated with other major cardiovascular anomalies. Lab Inves. 1957;6:44-64.

14. Khan MS, Bryant R 3rd, Kim SH, et al. Contemporary Outcomes of Surgical Repair of Total Anomalous Pulmonary Venous Connection in Patients With Heterotaxy Syndrome. Ann Thorac Surg. 2015;99:2134-2139.

15. Karaci AR, Harmandar B, Aydemir NA, et al. Early and intermediate term results for surgical correction of total anomalous pulmonary venous connection. J Card Surg. 2012;27:376-380.

16. Yong MS, d' Udekem Y, Robertson T, et al. Outcomes of surgery for simple total anomalous pulmonary venous drainage in neonates. Ann Thorac Surg. 2011;91:1921-1927.

17. Husain SA, Maldonado E, Rasch D, et al. Total Anomalous Pulmonary Venous Connection: factors associated with mortality and recurent pulmonary venous obstruction. Ann Thorac Surg. 2012;94:825-314.

18. Tanel RE, Kirshbom PM, Paridon SM, et al. Long-term noninvasive arrhythmia assessment after total anomalous pulmonary venous connection repair. Am Heart J. 2007;153:267-274. 
19. Milovanović V, Mimić B, Vulićević I, et al. Outcomes of surgery for total anomalous pulmonary venous drainage. Srp Arh Celok Lek. 2014;142:164-169.

20. Hoashi T, Kagisaki K, Oda T, et al. Long-term results of treatments for functional single ventricle associated with extracardiac type total anomalous pulmonary venous connection. Eur J Cardiothorac Surg. 2013;43:965-970
21. Yoshimura N, Fukahara K, Yamashita A, et al. Current topics in surgery for isolated total anomalous pulmonary venous connection. Surg Today. 2014:44:2221-2226.

22. Korbmacher B, Büttgen S, Schulte HD, et al. Long-term results after repair of total anomalous pulmonary venous connection. Thorac Cardiovasc Surg. 2001;49:101-106. 